\title{
New Persistent Opioid and Benzodiazepine Use After Curative-Intent Treatment in Patients With Breast Cancer
}

\author{
Mandy R. Sakamoto, MD; Megan Eguchi, MPH²; Christine M. Azelby, MD; Jennifer R. Diamond, MD;
} Christine M. Fisher, MD"; Virginia F. Borges, MD³; Cathy J. Bradley, $\mathrm{PhD}^{2}$; and Peter Kabos, $\mathrm{MD}^{3}$

\begin{abstract}
Background: Opioid and benzodiazepine use and abuse is a national healthcare crisis to which patients with cancer are particularly vulnerable. Long-term use and risk factors for opioid and benzodiazepine use in patients with breast cancer is poorly characterized. Methods: We conducted a retrospective population-based study of patients with breast cancer diagnosed between 2008 and 2015 undergoing curative-intent treatment identified through the SEERMedicare linked database. Primary outcomes were new persistent opioid use and new persistent benzodiazepine use. Factors associated with new opioid and benzodiazepine use were investigated by univariate and multivariable logistic regression. Results: Among opioidnaïve patients, new opioid use was observed in 22,418 (67.4\%). Of this group, $611(2.7 \%)$ developed persistent opioid use at 3 months and $157(0.7 \%)$ at 6 months after treatment. Risk factors for persistent use at 3 and 6 months included stage III disease (odds ratio [OR], 2.16; $95 \% \mathrm{Cl}, 1.49-3.12$, and $\mathrm{OR}, 3.48 ; 95 \% \mathrm{Cl}, 1.58-7.67)$, surgery plus chemotherapy $(\mathrm{OR}, 1.44 ; 95 \% \mathrm{Cl}, 1.10-1.88$, and $\mathrm{OR}, 2.28 ; 95 \% \mathrm{Cl}$, 1.40-3.71), surgery plus chemoradiation therapy $(\mathrm{OR}, 1.47 ; 95 \% \mathrm{Cl}$, $1.10-1.96$, and $\mathrm{OR}, 2.34 ; 95 \% \mathrm{Cl}, 1.38-3.96)$, and initial tramadol use (OR, 2.66; 95\% Cl, 2.05-3.46, and OR, 3.12; 95\% Cl, 1.93-5.04). Among benzodiazepine-naïve patients, new benzodiazepine use was observed in 955 (10.3\%), and 111 (11.6\%) developed new persistent use at 3 months. Tamoxifen use was statistically significantly associated with new persistent benzodiazepine use at 3 months. Conclusions: A large percentage of patients receiving curative-intent treatment of breast cancer were prescribed new opioids; however, only a small number developed new persistent opioid use. In contrast, a smaller proportion of patients received a new benzodiazepine prescription; however, new persistent use after completion of treatment was more likely and particularly related to concurrent treatment with tamoxifen.
\end{abstract}

J Natl Compr Canc Netw 2021;19(1):29-38 doi: $10.6004 /$ jnccn.2020.7612

${ }^{1}$ Department of Medicine, ${ }^{2}$ Department of Health Systems, Management, and Policy, ${ }^{3}$ Division of Medical Oncology, Department of Medicine, and

${ }^{4}$ Department of Radiation Oncology, University of Colorado Anschutz Medical Campus, Aurora, Colorado.

See JNCCN.org for supplemental online content.

\section{Background}

Management of pain and distress is a critical aspect of cancer care with significant impact on quality of life. ${ }^{1,2}$ Among patients with breast cancer, $25 \%$ to $89 \%$ experience pain along the disease trajectory and $25 \%$ to $60 \%$ report chronic pain related to cancer treatment. ${ }^{3-5}$ Psychologic distress affects up to $50 \%$ of survivors of breast cancer and may lead to increased consumption of various psychotropic medications. ${ }^{6-8}$ Opioids remain the mainstay treatment of cancer pain, placing patients at risk for misuse, dependence, and other adverse effects. ${ }^{9}$ These risks amplify with prolonged use, especially when taken in combination with sedating medications and in older patients (aged $>65$ years). ${ }^{10-12}$

Current guidelines for prescribing opioids in patients with cancer focus on relieving acute pain or pain associated with advanced disease. ${ }^{13-16}$ A recent study of patients with cancer undergoing curative-intent surgery found that $10.4 \%$ had new persistent opioid use at 1 year, highlighting the need to minimize prolonged opioid treatment in the absence of active disease. ${ }^{17}$ Furthermore, although providers are cautioned against coprescribing benzodiazepines and opioids, the benzodiazepine lorazepam is an approved and frequently prescribed adjunct to antiemetic drugs for chemotherapy-related nausea. ${ }^{18}$ Few studies have evaluated short- and long-term opioid use in patients with breast cancer and are limited to single treatment modalities or descriptive statistics. Dispensing patterns of benzodiazepines in patients with breast cancer remain poorly characterized.

Given the prevalence of treatment-related pain and psychosocial consequences after a diagnosis of breast cancer, we hypothesize that opioid and benzodiazepine use after treatment remains high. We performed a retrospective cohort analysis using population-based data to evaluate new and persistent opioid and benzodiazepine

See page 119 for related commentary. 
use and risk factors among patients with breast cancer who received curative-intent treatment.

\section{Methods}

\section{Data Source}

This study used data from the SEER-Medicare linked database from 2008 through 2015. This dataset represents $28 \%$ of the US population and contains demographic, clinical, mortality, and medication prescription information on Medicare beneficiaries with cancer. ${ }^{19}$ This project was reviewed by the Colorado Multiple Institutional Review Board and deemed exempt because all data were deidentified.

\section{Study Sample and Definitions}

We selected female patients aged $\geq 66$ years with stage 0 -III first primary breast cancer diagnosed in 2008 through 2015. Supplemental eFigure 1 illustrates the study selection criteria (available with this article at JNCCN.org). We limited our sample to patients aged $\geq 66$ years to obtain 12 months of claims data prior to diagnosis. We included patients undergoing the following curative-intent treatments: surgery, surgery plus radiation therapy (RT), surgery plus chemotherapy, and surgery plus chemoradiation therapy (CRT). Surgery was defined as lumpectomy or mastectomy. We required that treatment be initiated within 6 months of diagnosis, last no more than 6 months, and no additional treatments be initiated in the 12 weeks after treatment. Patients had to be continuously enrolled in Medicare Parts $\mathrm{A}, \mathrm{B}$, and D during a 12-month period before and after treatment initiation to ensure complete claims data.

We included patients who were opioid- and benzodiazepine-naïve, defined as those who did not fill opioid or benzodiazepine prescriptions during the 12 months through 31 days preceding treatment initiation. This approach is well described in previous studies of surgical patients and accounts for preoperative opioid prescriptions intended for postoperative use. ${ }^{17,20-22}$ For opioid analyses, we included patients diagnosed in 2008 through 2015 who were opioid- and benzodiazepinenaïve. However, prior benzodiazepine prescriptions could only be identified for patients diagnosed in 2014 through 2015 because benzodiazepine use was limited to Part D claims beginning in $2013^{23}$; therefore, benzodiazepine and concurrent utilization analyses were limited to patients diagnosed in 2014 through 2015. Patients with other malignancy, stage IV disease, enrolled in hospice, or with $<6$ months survival after treatment were excluded.

\section{Outcomes}

This study had 2 primary outcomes: new opioid use, defined as previously opioid-naïve patients who filled at least one opioid prescription between the start of treatment and 3 months after the end of treatment, and new persistent opioid use at 3 and 6 months after the end of treatment. Medication use was considered persistent if the time between the last day of one prescription and the first day of the next prescription was $<30$ days, as described in prior literature. ${ }^{24,25}$ We examined new benzodiazepine use in benzodiazepine-naïve patients using similar definitions. Due to limited data on benzodiazepine prescriptions, persistent benzodiazepine use was reported at 3 months only. Finally, we examined new concomitant opioid and benzodiazepine use, defined as overlap of prescriptions by at least 1 day from the start of treatment through 6 months after the end of treatment.

We used National Drug Codes and generic names to identify opioids and benzodiazepines reported in Part D prescription drug claims, and determined the time taking opioids and benzodiazepines based on prescription fill dates and the number of days' supply dispensed. Opioids and benzodiazepines included in the analysis are shown in supplemental eTable1.

\section{Patient Characteristics}

We used SEER data on patient age, year of diagnosis, race/ethnicity, marital status, poverty rate at the census tract, education level, practice setting, census tract ruralurban commuting area codes, geographic region, and tumor stage (Table 1). Part D claims were used to determine the first opioid prescribed. Claims from the year prior to diagnosis were used to determine number of comorbid conditions and history of depression or anxiety before treatment initiation.

\section{Statistical Analysis}

We performed chi-square tests to evaluate univariate associations with initial and persistent use of the medications of interest and used logistic regression to obtain multivariate estimates. Only patients with new medication use were included in analyses examining persistent use. We used the Kaplan-Meier method to determine median opioid and benzodiazepine prescription length after the end of treatment. We reported prescription length in weeks and censored patients with continued use beyond 6 months. We evaluated statistical significance at $P<.05$ and performed all analyses using SAS 9.4 (SAS Institute Inc). Observations with missing values were not included in the logistic analysis $(n=24)$.

\section{Results}

We identified 33,288 patients with breast cancer (stages 0 -III) who were opioid-naïve at diagnosis and received curative-intent treatment in 2008 through 2015. The average age in the study population was 75.8 years. Most patients were White, unmarried, living in urban areas in the western part of the United States, and in census tracts 
Table 1. Characteristics of Opioid-Naiive Patients

\begin{tabular}{|c|c|c|c|c|}
\hline Characteristic & $\begin{array}{c}\text { No Opioid Use } \\
n(\%)\end{array}$ & $\begin{array}{c}\text { New Opioid Use } \\
\text { n (\%) }\end{array}$ & $\begin{array}{c}\text { New Persistent Opioid Use } \\
\text { at } 3 \text { mo } \\
n(\%)\end{array}$ & $\begin{array}{l}\text { New Persistent Opioid Use } \\
\text { at } 6 \mathrm{mo}^{\mathrm{a}} \\
\mathrm{n}(\%)\end{array}$ \\
\hline Total, n & 10,856 & 22,418 & 611 & 157 \\
\hline \multicolumn{5}{|l|}{ Year of diagnosis } \\
\hline 2008-2010 & 4,494 (41.4) & $7,202(32.1)$ & $258(42.2)$ & $85(54.1)$ \\
\hline 2014-2015 & $2,785(25.6)$ & 6,464 (28.8) & $128(20.9)$ & $21(13.4)$ \\
\hline \multicolumn{5}{|l|}{ Race/Ethnicity } \\
\hline White/Non-Hispanic & $9,116(84.0)$ & $18,235(81.3)$ & $507(82.8)$ & $131(83.4)$ \\
\hline Black/Other & $1,740(16.0)$ & $4,197(18.7)$ & $105(17.2)$ & $26(16.6)$ \\
\hline \multicolumn{5}{|l|}{ Marital status } \\
\hline $0 \%-20 \%$ & $9,287(85.5)$ & $18,275(81.5)$ & $462(75.5)$ & $113(72.0)$ \\
\hline$>20 \%$ & $1,569(14.5)$ & $4,157(18.5)$ & $150(24.5)$ & $44(28.0)$ \\
\hline \multicolumn{5}{|l|}{$\%$ with $\leq$ high school education } \\
\hline Below median (more educated) & $6,000(55.3)$ & $11,232(50.1)$ & $256(41.8)$ & $59(37.6)$ \\
\hline Above median (less educated) & $4,856(44.7)$ & $11,200(49.9)$ & $356(58.2)$ & $98(62.4)$ \\
\hline \multicolumn{5}{|l|}{ Practice setting } \\
\hline Nonteaching hospital & $5,081(46.8)$ & $10,470(46.7)$ & $323(52.8)$ & $84(53.5)$ \\
\hline Teaching hospital & $5,775(53.2)$ & $11,962(53.3)$ & $289(47.2)$ & $73(46.5)$ \\
\hline \multicolumn{5}{|l|}{ Patient location } \\
\hline West & $4,670(43.0)$ & $9,343(41.6)$ & $250(40.9)$ & $63(40.1)$ \\
\hline \multicolumn{5}{|l|}{ Tumor stage ${ }^{b}$} \\
\hline 0 (DCIS) & $2,291(21.1)$ & $4,045(18.0)$ & $75(12.3)$ & $11(7.0)$ \\
\hline 1 & $5,505(50.7)$ & $11,269(50.2)$ & $277(45.3)$ & $58(36.9)$ \\
\hline ॥ & $2,563(23.6)$ & $5,799(25.9)$ & $182(29.7)$ & $62(39.5)$ \\
\hline III & $497(4.6)$ & $1,319(5.9)$ & 78 (12.7) & $26(16.6)$ \\
\hline \multicolumn{5}{|l|}{ Comorbidity index score } \\
\hline 0 & $6,480(59.7)^{\star}$ & $13,293(59.3)^{\star}$ & 287 (46.9) & $64(40.8)$ \\
\hline 1 & $2,492(23.0)$ & $5,396(24.0)$ & $162(26.5)$ & $55(35.0)$ \\
\hline$\geq 2$ & $1,884(17.3)$ & $3,743(16.7)$ & $163(26.6)$ & $38(24.2)$ \\
\hline \multicolumn{5}{|l|}{ Prior depression or anxiety } \\
\hline No & $9,783(90.1)$ & $20,012(89.2)$ & $518(84.6)$ & $132(84.1)$ \\
\hline Yes & $1,073(9.9)$ & $2,420(10.8)$ & 94 (15.4) & 25 (15.9) \\
\hline
\end{tabular}




\begin{tabular}{|c|c|c|c|c|}
\hline Characteristic & $\begin{array}{c}\text { No Opioid Use } \\
n(\%)\end{array}$ & $\begin{array}{c}\text { New Opioid Use } \\
n(\%)\end{array}$ & $\begin{array}{c}\text { New Persistent Opioid Use } \\
\text { at } 3 \mathrm{mo} \\
\text { n (\%) }\end{array}$ & $\begin{array}{c}\text { New Persistent Opioid Use } \\
\text { at } 6 \mathrm{mo}^{\mathrm{a}} \\
\mathrm{n}(\%)\end{array}$ \\
\hline \multicolumn{5}{|l|}{ Treatment type } \\
\hline Surgery alone & $5,166(47.6)$ & $8,212(36.6)$ & $221(36.1)$ & $47(29.9)$ \\
\hline Surgery + RT & $4,477(41.2)$ & $9,763(43.5)$ & $211(34.5)$ & $45(28.7)$ \\
\hline Surgery + chemotherapy & $646(6.0)$ & $2,472(11.0)$ & $103(16.8)$ & $38(24.2)$ \\
\hline Surgery + CRT & $567(5.2)$ & $1,985(8.9)$ & 77 (12.6) & $27(17.2)$ \\
\hline \multicolumn{5}{|l|}{ Endocrine therapy type } \\
\hline \multicolumn{5}{|l|}{ Aromatase inhibitors } \\
\hline No & $5,097(47.0)$ & $9,031(40.3)$ & $232(37.9)$ & $56(35.7)$ \\
\hline Yes & $5,759(53.0)$ & $13,401(59.7)$ & $380(62.1)$ & $101(64.3)$ \\
\hline \multicolumn{5}{|l|}{ Tamoxifen } \\
\hline No & $9,055(83.4)$ & $18,603(82.9)$ & $517(84.5)$ & $124(79.0)$ \\
\hline Yes & $1,801(16.6)$ & $3,829(17.1)$ & $95(15.5)$ & $33(21.0)$ \\
\hline \multicolumn{5}{|l|}{ First opioid prescribed } \\
\hline Hydrocodone & - & $13,537(60.4)$ & $367(60.0)$ & $98(62.4)$ \\
\hline Oxycodone & - & $5,387(24.0)$ & $109(17.8)$ & $23(14.7)$ \\
\hline Tramadol & - & $1,180(5.3)$ & $75(12.2)$ & $22(14.0)$ \\
\hline Other ${ }^{c}$ & - & $2,328(10.3)$ & $61(10.0)$ & $14(8.9)$ \\
\hline
\end{tabular}

Bold indicates statistically significant estimates based on a $P$ value threshold of .05 on the univariate analysis.

Abbreviations: CRT, chemoradiation therapy; DCIS, ductal carcinoma in situ; RT, radiation therapy.

aPatients in the 6-month group are also included in the 3-month group.

bStaging based on the 6th edition of AJCC Cancer Staging Manual.

Includes 115 patients who filled $>1$ opioid; however, numbers were too small to report combination use at 3 and 6 months.

${ }^{\star} P=.05-.06$

with $\leq 20 \%$ of the population below the poverty level. Of patients undergoing curative-intent treatment in 2014 through 2015, a total of 9,249 were both opioid- and benzodiazepine-naïve.

\section{New Opioid Use}

Among previously opioid-naïve patients, 22,418 (67.4\%) filled a new opioid prescription during the treatment period (Table 1), and 611 (2.7\%) of those patients developed new persistent opioid use through 3 months and $157(0.7 \%)$ through 6 months after treatment. Median and mean time taking opioids was 0.7 and 1.9 weeks, respectively. New opioid users were more likely to receive multimodality treatment and aromatase inhibitors, and persistent opioid users were more likely to receive multimodality treatment, have higher stages of disease, and higher Charlson comorbidity index scores than patients with no opioid use.

We then estimated the odds ratios (ORs) for likelihood of new opioid use (Table 2). Patients with higher stages of disease were more likely to receive a new opioid prescription, but only patients with stage III disease were more likely to have continued use at 3 and 6 months (OR, 2.16 [95\% CI, 1.49-3.12] and 3.48 [95\% CI, 1.58-7.67], respectively). Patients who underwent surgery plus chemotherapy or surgery plus CRT were more likely to fill a new opioid prescription (OR, 1.94 [95\% CI, 1.75-2.14] and 1.79 [95\% CI, 1.61-1.99], respectively) and remain on opioids at 3 months (OR, 1.44 [95\% CI, 1.10-1.88] and 1.47 [95\% CI, 1.10-1.96], respectively) and 6 months (OR, 2.28 [95\% CI, $1.40-3.71$ ] and 2.34 [95\% CI, 1.38-3.96], respectively) compared with those undergoing surgery alone.

The most commonly prescribed opioids included hydrocodone $(60.5 \%)$, oxycodone $(17.8 \%)$, and tramadol (12.2\%). Compared with initial hydrocodone use, initial tramadol use was more likely to be associated with continued opioid use at 3 and 6 months (OR, 2.66 [95\% CI, 2.05-3.46] and 3.12 [95\% CI, 1.93-5.04], respectively). Overall, persistent opioid use at 3 and 6 months significantly decreased from 2008 to 2015 (OR, 0.90 [95\% CI, 0.87-0.94] and 0.80 [95\% CI, $0.75-0.87]$, respectively).

\section{New Benzodiazepine Use}

Among opioid- and benzodiazepine-naïve patients diagnosed in 2014 through 2015, 955 (10.3\%) filled a new benzodiazepine prescription during the treatment period, and $111(11.6 \%)$ of those patients developed new persistent benzodiazepine use 3 months after treatment (eTable 2). Median and mean time on benzodiazepines 
Table 2. Logistic Regression Models for New Opioid Use

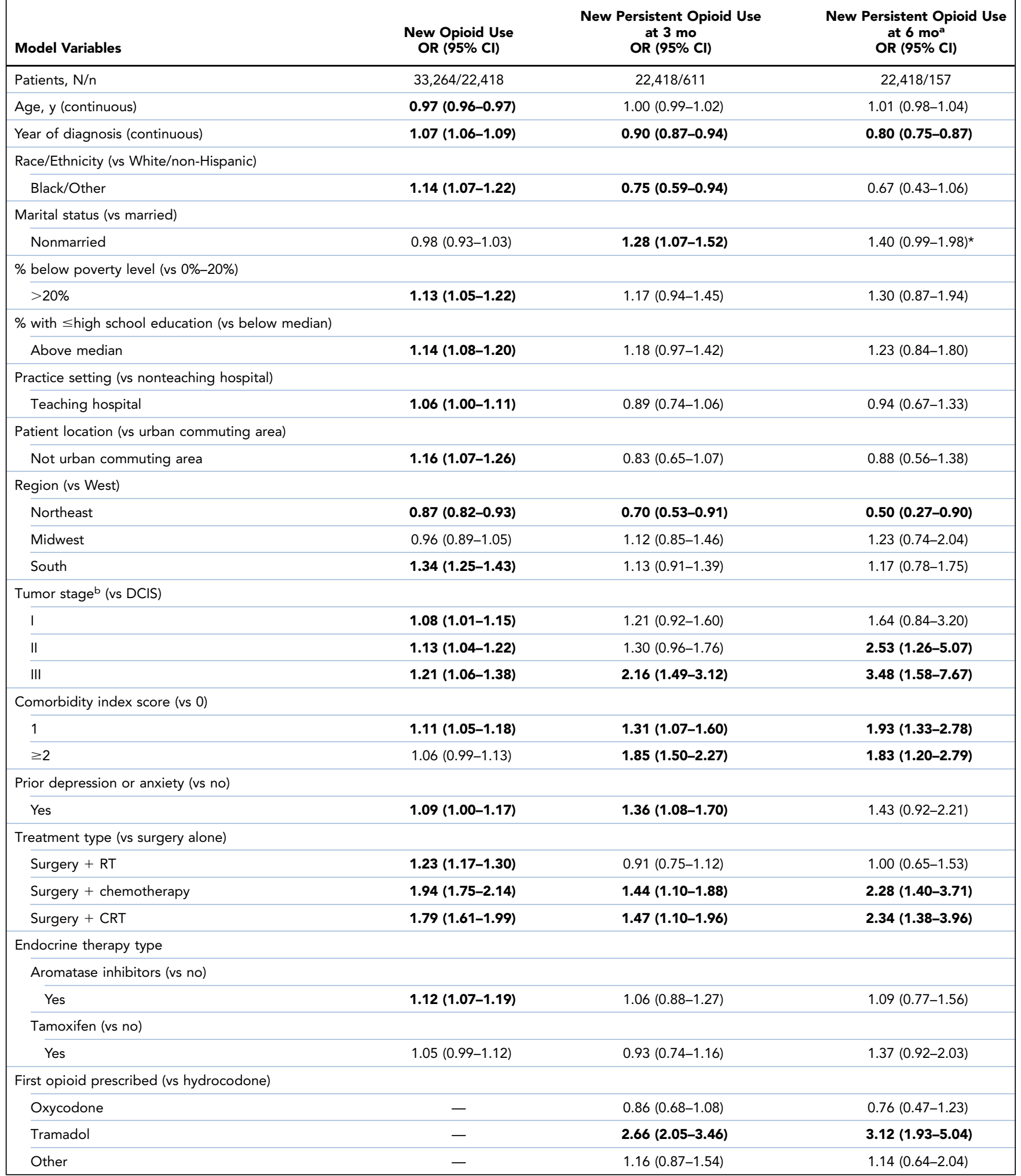

Bold indicates statistically significant estimates based on a $P$ value threshold of .05 on the univariate analysis.

Abbreviations: CRT, chemoradiation therapy; DCIS, ductal carcinoma in situ; OR; odds ratio; RT, radiation therapy.

aPatients in the 6-month group are also included in the 3-month group.

bStaging based on the 6th edition of AJCC Cancer Staging Manual.

$\star_{P}=.05-.06$ 
was 2.4 and 3.4 weeks, respectively. Patients who underwent surgery plus chemotherapy or surgery plus CRT were 4 times as likely to fill a new benzodiazepine prescription (OR, 4.05 [95\% CI, 3.23-5.08] and 4.03 [95\% CI, 3.21-5.07], respectively). New benzodiazepine use was also positively associated with depression/anxiety, advanced stages of disease, and negatively associated with nonmarried status, lower education, treatment at teaching hospitals, and Midwestern and Southern regions (Table 3). Patients who received tamoxifen or with depression/anxiety were more likely to develop persistent benzodiazepine use at 3 months (OR, 1.93 [95\% CI, 1.12-3.33] and 1.74 [CI, 1.01-3.01], respectively). The other subgroup consisted primarily of clonazepam and temazepam, and was associated with $>4.5$-fold increased risk of persistent benzodiazepine use; however, medications could not be considered separately because of small sample size. There was no difference in persistent benzodiazepine use from 2014 to 2015 .

\section{Concomitant Opioid and Benzodiazepine Use}

Among opioid- and benzodiazepine-naïve patients diagnosed in 2014 through 2015, 370 (4.0\%) filled new prescriptions for both during the observation period (eTable 2). Concurrent use was positively associated with advanced stages of disease, surgery plus chemotherapy, and surgery plus CRT, and negatively associated with increasing age, non-White race, and Midwestern and Southern regions (Table 3).

\section{Discussion}

We present the largest study to date of opioid and benzodiazepine use in patients with breast cancer who underwent curative-intent treatment. Opioids were used by most patients during the treatment period; however, few developed new persistent opioid use. Long-term opioid use was associated with higher stages of disease, higher number of comorbidities, multimodality regimens, and type of initial opioid prescribed. Although a smaller percentage of patients received a new benzodiazepine, they were more likely to develop persistent use after treatment. Prior depression/anxiety, use of tamoxifen, and select benzodiazepines were the only treatment- or patient-related factors associated with long-term benzodiazepine use.

Almost two-thirds of opioid-naïve patients filled at least one opioid prescription. To our knowledge, only one prior study quantified the prevalence of opioid use among patients with breast cancer who received a similar combination of treatments. ${ }^{7}$ Although this study reported a lower rate of opioid use during the active treatment phase (25\%), data were obtained from 1998 to 2011, during which they observed a dramatic rise in opioid use from $9 \%$ to $41 \%$, respectively. This suggests that opioid use has continued to increase, possibly because of greater acceptance of opioids for the treatment of pain and concerns related to undertreatment of cancer pain. ${ }^{26-28}$ Our results are more comparable to the $57 \%$ of patients with breast cancer who received opioids during adjuvant endocrine therapy ${ }^{29}$ and the $31 \%$ to $45 \%$ during curativeintent chemotherapy. ${ }^{30}$ We also identified several treatment- and patient-related factors that were predictive of new opioid use. Aromatase inhibitors were associated with short-term opioid use, whereas tamoxifen was not. This finding differs from those of a recent population-based study that showed no significant relationship between adjuvant endocrine therapy type and opioid use. ${ }^{29}$

Our study showed that a small proportion of patients developed new persistent opioid use at 3 months $(2.7 \%)$ and 6 months $(<1 \%)$. These numbers are lower than the $10.4 \%$ reported in a recent study by Lee et $\mathrm{al}^{17}$ of patients with various cancer types undergoing curative-intent surgery. The study defined new persistent opioid use as previously opioid-naïve patients who filled an opioid prescription attributed to surgery and an additional opioid prescription between 90 and 180 days after surgery; however, adjuvant chemotherapy often extends beyond 90 days postoperatively, which may explain the differences. Patients in the study were also younger, which is an independent risk factor for opioid use..$^{31,32}$ Similar to the study by Lee et al, ${ }^{17}$ our results demonstrated an increased likelihood of persistent opioid use when surgery was combined with chemotherapy with or without radiation therapy (RT). Although the combination of surgery and RT was associated with increased short-term opioid use, there was no significant association with long-term use. This may be because of advances in RT that provide more targeted and less painful treatment, whereas chemotherapy-induced pain remains a challenge..$^{33-35}$

Initial use of tramadol was associated with long-term opioid use, which is similar to findings reported in patients without cancer. ${ }^{36}$ Despite the low percentage of patients prescribed tramadol, our results suggest that a choice of a different opiate should be considered. History of depression/anxiety, number of comorbidities, and advanced disease have been shown to contribute to opioid use in patients with breast cancer ${ }^{22}$; our study adds to this literature by better understanding the association with persistent opioid use after treatment, which may be the more relevant patient outcome. Although new short-term opioid use in patients with breast cancer may be increasing, our findings suggest that longterm opioid use has declined over time.

Approximately $10 \%$ of patients received a new benzodiazepine and $12 \%$ developed persistent use at 3 months after treatment. Our results are comparable to those from a large population-based study of patients with incident nonmetastatic breast cancer in which 
Table 3. Logistic Regression Models for New BZP Use and Concomitant Opioid and BZP Use

\begin{tabular}{|c|c|c|c|}
\hline Model Variables & $\begin{array}{l}\text { New BZP Use } \\
\text { OR }(95 \% \mathrm{Cl})\end{array}$ & $\begin{array}{c}\text { New Persistent BZP Use } \\
\text { at } 3 \mathrm{mo} \\
\text { OR }(95 \% \mathrm{Cl})\end{array}$ & $\begin{array}{c}\text { New Opioid and BZP Use } \\
\text { OR }(95 \% \mathrm{Cl})\end{array}$ \\
\hline Patients, N/n & $9,244 / 955$ & $955 / 11$ & $9,244 / 370$ \\
\hline Year of diagnosis (continuous) & $0.99(0.86-1.14)$ & $0.82(0.53-1.26)$ & $1.05(0.85-1.31)$ \\
\hline \multicolumn{4}{|c|}{ Race/Ethnicity (vs White/non-Hispanic) } \\
\hline \multicolumn{4}{|l|}{ Marital status (vs married) } \\
\hline Nonmarried & $0.85(0.73-0.98)$ & $0.99(0.65-1.53)$ & $0.92(0.74-1.14)$ \\
\hline \multicolumn{4}{|c|}{$\%$ below poverty level (vs $0 \%-20 \%$ ) } \\
\hline$>20 \%$ & $0.91(0.72-1.14)$ & $0.95(0.49-1.83)$ & $0.85(0.61-1.20)$ \\
\hline \multicolumn{4}{|c|}{$\%$ with $\leq$ high school education (vs below median) } \\
\hline \multicolumn{4}{|c|}{ Patient location (vs urban commuting area) } \\
\hline Not urban commuting area & $1.10(0.87-1.40)$ & $1.13(0.57-2.24)$ & $1.33(0.95-1.86)$ \\
\hline \multicolumn{4}{|l|}{ Region (vs West) } \\
\hline Northeast & $1.04(0.86-1.26)$ & $0.94(0.53-1.68)$ & $1.03(0.77-1.37)$ \\
\hline Midwest & $0.69(0.54-0.90)$ & $1.02(0.48-2.17)$ & $0.63(0.43-0.94)$ \\
\hline South & $0.74(0.60-0.91)$ & $1.59(0.88-2.85)$ & $0.71(0.52-0.97)$ \\
\hline \multicolumn{4}{|l|}{ Tumor stage ${ }^{a}$ (vs DCIS) } \\
\hline I & $1.31(1.03-1.67)$ & $0.77(0.40-1.45)$ & $1.37(0.96-1.95)$ \\
\hline$\|$ & $1.96(1.51-2.54)^{b}$ & $0.53(0.25-1.09)^{b}$ & $1.76(1.19-2.61)$ \\
\hline \multicolumn{4}{|c|}{ Treatment type (vs surgery alone) } \\
\hline Surgery + RT & $0.87(0.72-1.05)$ & $1.12(0.65-1.94)$ & $0.54(0.41-0.70)$ \\
\hline Surgery + chemotherapy & $4.05(3.23-5.08)$ & $1.48(0.77-2.84)$ & $1.76(1.26-2.46)$ \\
\hline Surgery + CRT & $4.03(3.21-5.07)$ & $0.63(0.29-1.38)$ & $1.46(1.03-2.07)$ \\
\hline \multicolumn{4}{|l|}{ Endocrine therapy type } \\
\hline \multicolumn{4}{|l|}{ Aromatase inhibitors (vs no) } \\
\hline Yes & $1.08(0.92-1.28)$ & $0.89(0.56-1.42)$ & $1.16(0.90-1.48)$ \\
\hline \multicolumn{4}{|l|}{ Tamoxifen (vs no) } \\
\hline Yes & $0.93(0.75-1.16)$ & $1.93(1.12-3.33)$ & $0.98(0.71-1.36)$ \\
\hline \multicolumn{4}{|c|}{ First BZP prescribed (vs lorazepam) } \\
\hline Alprazolam & - & $1.37(0.80-2.34)$ & - \\
\hline Diazepam & - & $0.93(0.47-1.84)$ & - \\
\hline Other & - & $4.32(2.18-8.58)$ & - \\
\hline
\end{tabular}

Bold indicates statistically significant estimates based on a $P$ value threshold of .05 on the univariate analysis.

Abbreviations: BZP, benzodiazepine; CRT, chemoradiation therapy; DCIS, ductal carcinoma in situ; RT, radiation therapy.

aStaging based on 6th edition of the AJCC Cancer Staging Manual.

bStages II and III were combined to ensure cell size $>11$ for patient confidentiality. 
$17.5 \%$ developed new benzodiazepine use during active care (defined as the 12 months after diagnosis of cancer) and $12.4 \%$ during first-year survivorship. ${ }^{7}$ Another study found that $7.9 \%$ of survivors of breast cancer were prescribed benzodiazepines for a median of 14 years from time of diagnosis to the last benzodiazepine prescription..$^{37}$ In contrast, a single-center study showed $33 \%$ of patients with breast cancer remained on benzodiazepines 90 days after curative chemotherapy; however, sample size was small, patients were younger, and those with prior benzodiazepine use were not excluded. ${ }^{30}$

Risk factors for benzodiazepine use during and after breast cancer treatment have not been previously reported. Multimodality treatment that includes surgery combined with chemotherapy with or without RT was associated with a 4-fold increased risk of benzodiazepine use during the treatment period. This is not unexpected because chemotherapy is highly emetogenic and recent guidelines support the use of benzodiazepines as adjunctive treatment. ${ }^{18}$ Patients receiving tamoxifen were almost twice as likely to continue benzodiazepines 3 months after treatment. Providers may favor prescribing benzodiazepines because of data suggesting that certain selective serotonin reuptake inhibitors (SSRIs) increase the risk of breast cancer recurrence when used concurrently with tamoxifen..$^{38-41}$ For example, paroxetine, fluoxetine, and sertraline are potent inhibitors of CYP2D6, an enzyme needed to convert tamoxifen into its active form, and are known to reduce the clinical efficacy of tamoxifen. Less potent inhibitors of CYP2D6, including citalopram and escitalopram, were not associated with an increased risk of recurrence, and noninhibitors of CYP2D6, including venlafaxine and mirtazapine, are thought to be at least equally as safe. Our results support that long-term benzodiazepine use is further driven by psychosocial distress, as anxiety and insomnia are the most commonly cited reasons for initiating and continuing benzodiazepines among breast cancer survivors. ${ }^{37}$ Lastly, although numbers for clonazepam and temazepam use are small, our results suggest that an alternative benzodiazepine should be considered.

We observed that $4 \%$ of patients received new concurrent opioids and benzodiazepines during the treatment period. Only one prior study reported the rate of opioid and benzodiazepine use in patients with cancer and curative disease. ${ }^{38}$ This was a small, single-center study of patients with breast cancer undergoing curative-intent chemotherapy that showed increased concomitant opioid and benzodiazepine use from $5 \%$ prior to starting treatment to $21 \%$ at the end of chemotherapy to $16 \%$ at 90 days after chemotherapy. In contrast, a 2016 report from the Centers for Medicare \& Medicaid Services showed that $8 \%$ of patients without cancer and not enrolled in hospice use opioids currently with benzodiazepines. ${ }^{42}$ Other studies in patients without cancer report differing percentages based on selected patient population and varying definitions of concomitancy. ${ }^{43-48}$

Finally, there is a substantial body of literature documenting the undertreatment of pain, including cancer pain, among racial and ethnic minorities. ${ }^{49-53}$ Our findings show that non-White patients were more likely to receive a new opioid during treatment, but significantly less likely to receive opioids at 3 months after treatment. These disparities may be partly explained by financial and environmental barriers experienced by minority groups that impair long-term pain management. ${ }^{53}$ Furthermore, although non-White patients were less likely to receive an opioid plus benzodiazepine, we found no difference between racial groups regarding receipt of benzodiazepines alone. We speculate that patients may be more comfortable discussing anxiety and nausea than pain symptoms with their doctor, given that fear of reporting pain and failure of providers to initiate conversations about pain are common barriers to opioid prescriptions and effective pain management in minority cancer survivors. ${ }^{53,54}$

This study has several limitations. First, patients were aged $\geq 66$ years, resulting in an older population compared with the national median age of 62 years for patients with breast cancer. ${ }^{55}$ Second, opioid and benzodiazepine use was determined by dispensing patterns, thus does not evaluate whether medications were actually consumed. Medications prescribed but not filled were not accounted for and may explain the subset of patients who did not receive prescriptions postoperatively. We were unable to determine indications for opioid and benzodiazepine use, and it is possible that prescriptions were provided for symptoms unrelated to a patient's cancer diagnosis. The sample size was too small to assess persistent benzodiazepine use at 6 months after treatment. Lastly, we did not evaluate opioid strength or cumulative dosing.

\section{Conclusions}

We found that $67 \%$ of opioid-naive patients with breast cancer receive opioids during curative-intent treatment. Although a small proportion of patients developed persistent use at 3 and 6 months, on a population level this translates to approximately 8,775 and 2,275 women per year, respectively. Only $10 \%$ of benzodiazepine-naïve patients with breast cancer receive benzodiazepines during treatment; however, persistent use at 3 months remains high, translating to $>37,700$ women annually. Encouraging use of select SSRIs among patients taking tamoxifen may be one strategy to reduce long-term benzodiazepine use. These findings demonstrate the importance of identifying high-risk patients, educating 
providers on nonopioid and nonbenzodiazepine approaches, and counseling patients throughout all phases of care.

Submitted February 20, 2020; accepted for publication June 26, 2020.

Author contributions: Study concept and design: Sakamoto, Eguchi, Bradley, Kabos. Data acquisition and analysis: Eguchi, Bradley. Data interpretation: Sakamoto, Bradley, Kabos. Methodology: Sakamoto, Eguchi, Bradley, Kabos. Manuscript writing: Sakamoto. Manuscript review and editing: All authors.
Disclosures: The authors have disclosed that they have not received any financial consideration from any person or organization to support the preparation, analysis, results, or discussion of this article.

Funding: This project was supported by Population Health Shared Resource, University of Colorado Cancer Center, P30CA046934; NCl grant CA22955 (C.J.B.), and $\mathrm{NCl}$ grant CA20544 (P.K.).

Correspondence: Peter Kabos, MD, Division of Medical Oncology, Department of Medicine, University of Colorado Anschutz Medical Campus, 12801 East 17th Avenue, Aurora, CO 80045.

Email: Peter.Kabos@cuanschutz.edu; and

Cathy J. Bradley, PhD, Department of Health Systems, Management, and Policy, University of Colorado Comprehensive Cancer Center, 13001 East 17th Place, Mail Stop B119, Aurora, CO 80045. Email: Cathy.Bradley@cuanschutz.edu

\section{References}

1. Institute of Medicine (US) Committee on Psychosocial Services to Cancer Patients/Families in a Community Setting; Adler NE, Page AEK, eds. Cancer Care for the Whole Patient: Meeting Psychosocial Health Needs. Washington, DC: National Academies Press; 2008.

2. Mantyh PW. Cancer pain and its impact on diagnosis, survival and quality of life. Nat Rev Neurosci 2006;7:797-809.

3. Gärtner R, Jensen MB, Nielsen J, et al. Prevalence of and factors associated with persistent pain following breast cancer surgery. JAMA 2009; 302:1985-1992.

4. Runowicz CD, Leach CR, Henry NL, et al. American Cancer Society/ American Society of Clinical Oncology breast cancer survivorship care guideline. CA Cancer J Clin 2016;66:43-73.

5. Johannsen M, Christensen S, Zachariae R, et al. Socio-demographic treatment-related, and health behavioral predictors of persistent pain 15 months and 7-9 years after surgery: a nationwide prospective study of women treated for primary breast cancer. Breast Cancer Res Treat 2015; 152:645-658.

6. Kornblith $A B$, Ligibel J. Psychosocial and sexual functioning of survivors of breast cancer. Semin Oncol 2003;30:799-813.

7. Syrowatka A, Chang SL, Tamblyn R, et al. Psychotropic and opioid medication use in older patients with breast cancer across the care trajectory: a populationbased cohort study. J Natl Compr Canc Netw 2016;14:1412-1419.

8. de Bock GH, Musters RF, Bos HJ, et al. Psychotropic medication during endocrine treatment for breast cancer. Support Care Cancer 2012;20: 1533-1540.

9. Bohnert ASB, Valenstein M, Bair MJ, et al. Association between opioid prescribing patterns and opioid overdose-related deaths. JAMA 2011; 305:1315-1321

10. Dalal S, Bruera E. Pain management for patients with advanced cancer in the opioid epidemic era. Am Soc Clin Oncol Educ Book 2019;39:24-35.

11. By the 2019 American Geriatrics Society Beers Criteria Update Expert Panel. American Geriatrics Society 2019 updated AGS Beers Criteria for potentially inappropriate medication use in older adults. J Am Geriatr Soc 2019;67:674-694

12. van Strien AM, Koek HL, van Marum RJ, et al. Psychotropic medications, including short acting benzodiazepines, strongly increase the frequency of falls in elderly. Maturitas 2013;74:357-362.

13. Portenoy RK. Treatment of cancer pain. Lancet 2011;377:2236-2247.

14. Swarm RA, Youngwerth JM, Anghelescu DL, et al. NCCN Clinical Practice Guidelines in Oncology: Adult Cancer Pain. Version 1.2020. Accessed May 1, 2020. To view the most recent version, visit NCCN.org

15. Paice JA, Portenoy R, Lacchetti $C$, et al. Management of chronic pain in survivors of adult cancers: American Society of Clinical Oncology clinical practice guideline. J Clin Oncol 2016;34:3325-3345.

16. Lefkowits $C$, Buss MK, Ramzan AA, et al. Opioid use in gynecologic oncology in the age of the opioid epidemic: part I - effective opioid use across clinical settings, a society of gynecologic oncology evidence-based review. Gynecol Oncol 2018;149:394-400.

17. Lee JSJ, Hu HM, Edelman AL, et al. New persistent opioid use among patients with cancer after curative-intent surgery. J Clin Oncol 2017;35:4042-4049.

18. Hesketh PJ, Bohlke K, Kris MG. Antiemetics: American Society of Clinical Oncology clinical practice guideline update summary. J Oncol Pract 2017; 13:825-830.

19. SEER-Medicare: Description of SEER-Medicare Data Files. Accessed June 20, 2019. Available at: https://healthcaredelivery.cancer.gov/seermedicare/ overview/
20. Clarke H, Soneji N, Ko DT, et al. Rates and risk factors for prolonged opioid use after major surgery: population based cohort study. BMJ 2014; 348:g1251.

21. Brummett CM, Waljee JF, Goesling J, et al. New persistent opioid use after minor and major surgical procedures in US adults. JAMA Surg 2017 152:e170504

22. Marcusa DP, Mann RA, Cron DC, et al. Prescription opioid use among opioid-naive women Undergoing immediate breast reconstruction. Plas Reconstr Surg 2017;140:1081-1090.

23. Transition to Part $D$ coverage of benzodiazepines and barbiturates beginning in 2013. Published October 2, 2012. Accessed October 20, 2020 Available at: https://www.cms.gov/Medicare/Prescription-Drug-Coverage/PrescriptionDrugCovContra/HPMS-Guidance-History-Items/BenzoandBarbituratesin2013.html

24. McDermott JD, Eguchi M, Stokes WA, et al. Short- and long-term opioid use in patients with oral and oropharynx cancer. Otolaryngol Head Neck Surg 2019;160:409-419

25. Yaldo A, Wen L, Ogbonnaya A, et al. Opioid use among metastatic prostate cancer patients with skeletal-related events. Clin Ther 2016;38 1880-1889.

26. Manchikanti L, Fellows B, Ailinani H, et al. Therapeutic use, abuse, and nonmedical use of opioids: a ten-year perspective. Pain Physician 2010; 13:401-435

27. Bennett M, Paice JA, Wallace M. Pain and opioids in cancer care: benefits risks, and alternatives. Am Soc Clin Oncol Educ Book 2017; 37:705-713.

28. Deandrea S, Montanari M, Moja L, et al. Prevalence of undertreatment in cancer pain. A review of published literature. Ann Oncol 2008;19: 1985-1991.

29. Tan X, Camacho TF, LeBaron VT, et al. Opioid use among female breast cancer patients using different adjuvant endocrine therapy regimens. Breast Cancer Res Treat 2017; 165:455-465.

30. Yau WH, Roeland EJ, Revta C, et al. Opioid and benzodiazepine relative use in breast cancer patients before, during, and after curative chemotherapy. J Pain Palliat Care Pharmacother 2018;32:27-29.

31. Boscarino JA, Rukstalis M, Hoffman SN, et al. Risk factors for drug dependence among out-patients on opioid therapy in a large US health-care system. Addiction 2010;105:1776-1782.

32. Ciesielski $T$, lyengar $R$, Bothra $A$, et al. A tool to assess risk of de novo opioid abuse or dependence. Am J Med 2016;129:699-705.e4.

33. Hershman DL, Lacchetti C, Dworkin RH, et al. Prevention and management of chemotherapy-induced peripheral neuropathy in survivors of adult cancers: American Society of Clinical Oncology clinical practice guideline. J Clin Oncol 2014;32:1941-1967.

34. Kurita GP, Sjøgren P. Pain management in cancer survivorship. Acta Oncol 2015;54:629-634.

35. Chiu N, Zhang L, Dent R, et al. A prospective study of docetaxelassociated pain syndrome. Support Care Cancer 2018;26:203-211.

36. Shah A, Hayes CJ, Martin BC. Characteristics of initial prescription episodes and likelihood of long-term opioid use-United States, 2006-2015. MMWR Morb Mortal Wkly Rep 2017;66:265-269.

37. Vaidya R, Sood R, Karlin N, et al. Benzodiazepine use in breast cancer survivors: findings from a consecutive series of 1,000 patients. Oncology 2011;81:9-11.

38. Aubert RE, Stanek EJ, Yao J, et al. Risk of breast cancer recurrence in women initiating tamoxifen with CYP2D6 inhibitors [abstract]. J Clin Onco 2009;27(Suppl):Abstract CRA508. 
39. Kelly CM, Juurlink DN, Gomes T, et al. Selective serotonin reuptake inhibitors and breast cancer mortality in women receiving tamoxifen: a population based cohort study. BMJ 2010;340:c693.

40. Goetz MP, Kamal A, Ames MM. Tamoxifen pharmacogenomics: the role of CYP2D6 as a predictor of drug response. Clin Pharmacol Ther 2008;83: 160-166.

41. Sideras K, Ingle JN, Ames MM, et al. Coprescription of tamoxifen and medications that inhibit CYP2D6. J Clin Oncol 2010;28: 2768-2776.

42. Centers for Medicare and Medicaid Services Concurrent use of opioids and benzodiazepines in a Medicare Part D population. Published May 12, 2016. Accessed July 17, 2019. Available at: https://www.cms.gov/ Medicare/Prescription-Drug-Coverage/PrescriptionDrugCovContra/ Downloads/Concurrent-Use-of-Opioids-and-Benzodiazepines-in-aMedicare-Part-D-Population-CY-2015.pdf

43. Hwang CS, Kang EM, Kornegay CJ, et al. Trends in the concomitant prescribing of opioids and benzodiazepines, 2002-2014. Am J Prev Med 2016;51:151-160.

44. Agarwal SD, Landon BE. Patterns in outpatient benzodiazepine prescribing in the United States. JAMA Netw Open 2019;2: e187399.

45. Hawkins EJ, Malte CA, Grossbard JR, et al. Prevalence and trends of concurrent opioid analgesic and benzodiazepine use among Veterans Affairs patients with post-traumatic stress disorder, 2003-2011. Pain Med 2015;16:1943-1954

46. Sun EC, Dixit A, Humphreys K, et al. Association between concurrent use of prescription opioids and benzodiazepines and overdose: retrospective analysis. BMJ 2017;356:j760.
47. Zin CS, Ismail F. Co-prescription of opioids with benzodiazepine and other co-medications among opioid users: differential in opioid doses. J Pain Res 2017;10:249-257.

48. Hernandez I, He M, Brooks MM, et al. Exposure-response association between concurrent opioid and benzodiazepine use and risk of opioidrelated overdose in Medicare Part D beneficiaries. JAMA Netw Open 2018;1:e180919.

49. Hoffman KM, Trawalter S, Axt JR, Oliver MN. Racial bias in pain assessment and treatment recommendations, and false beliefs about biological differences between blacks and whites. Proc Natl Acad Sci USA 2016;113:4296-4301.

50. Lee P, Le Saux M, Siegel R, et al. Racial and ethnic disparities in the management of acute pain in US emergency departments: meta-analysis and systematic review. Am J Emerg Med 2019;37:1770-1777.

51. Meghani SH, Byun E, Gallagher RM. Time to take stock: a meta-analysis and systematic review of analgesic treatment disparities for pain in the United States. Pain Med 2012;13:150-174.

52. Shah AA, Zogg CK, Zafar SN, et al. Analgesic access for acute abdominal pain in the emergency department among racial/ethnic minority patients: a nationwide examination. Med Care 2015;53:1000-1009.

53. Stein KD, Alcaraz KI, Kamson C, et al. Sociodemographic inequalities in barriers to cancer pain management: a report from the American Cancer Society's Study of Cancer Survivor-II (SCS-II). Psychooncology 2016;25: 1212-1221.

54. Tait RC, Chibnall JT. Racial/ethnic disparities in the assessment and treatment of pain: psychosocial perspectives. Am Psychol 2014;69:131-141.

55. Facts CS. Female Breast Cancer. Accessed July 18, 2019. Available at: https://seer.cancer.gov/statfacts/html/breast.html

See JNCCN.org for supplemental online content. 
Supplemental online content for:

\section{New Persistent Opioid and Benzodiazepine Use After Curative-Intent Treatment in Patients With Breast Cancer}

Mandy R. Sakamoto, MD; Megan Eguchi, MPH; Christine M. Azelby, MD; Jennifer R. Diamond, MD; Christine M. Fisher, MD; Virginia F. Borges, MD; Cathy J. Bradley, PhD; and Peter Kabos, MD

J Natl Compr Canc Netw 2021;19(1):29-38

eFigure 1: Study Cohort and Sample Criteria

eTable 1: Opioids and Benzodiazepines Included in Analysis

eTable 2. Characteristics of Opioid- and Benzodiazepine-Naïve Patients 


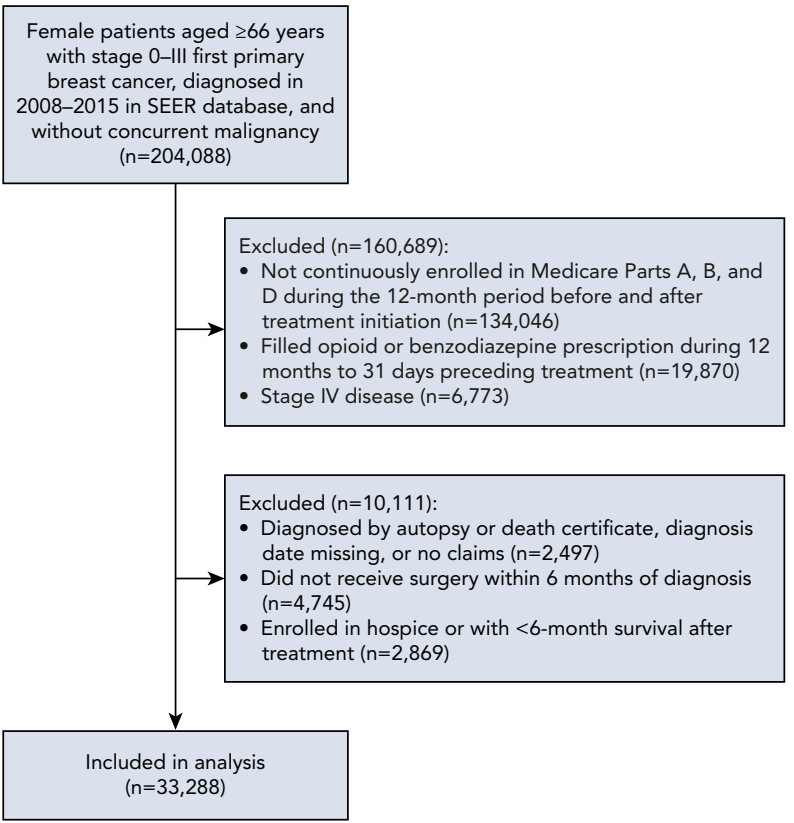

eFigure 1. Study cohort and sample criteria. 
2 - Sakamoto et al

eTable 1. Opioids and Benzodiazepines Included in Analysis

\begin{tabular}{|ll|}
\hline Opioids & Benzodiazepines \\
\hline Hydrocodone & Lorazepam \\
\hline Oxycodone & Alprazolam \\
\hline Tramadol & Diazepam \\
\hline Codeine & Clonazepam \\
\hline Fentanyl & Temazepam \\
\hline Hydromorphone & Chlordiazepoxide \\
\hline Meperidine & \\
\hline Morphine & \\
\hline Butorphanol & \\
\hline Pentazocine & \\
\hline Tapentadol & \\
\hline
\end{tabular}




\section{eTable 2. Characteristics of Opioid- and Benzodiazepine-Naïve Patients}

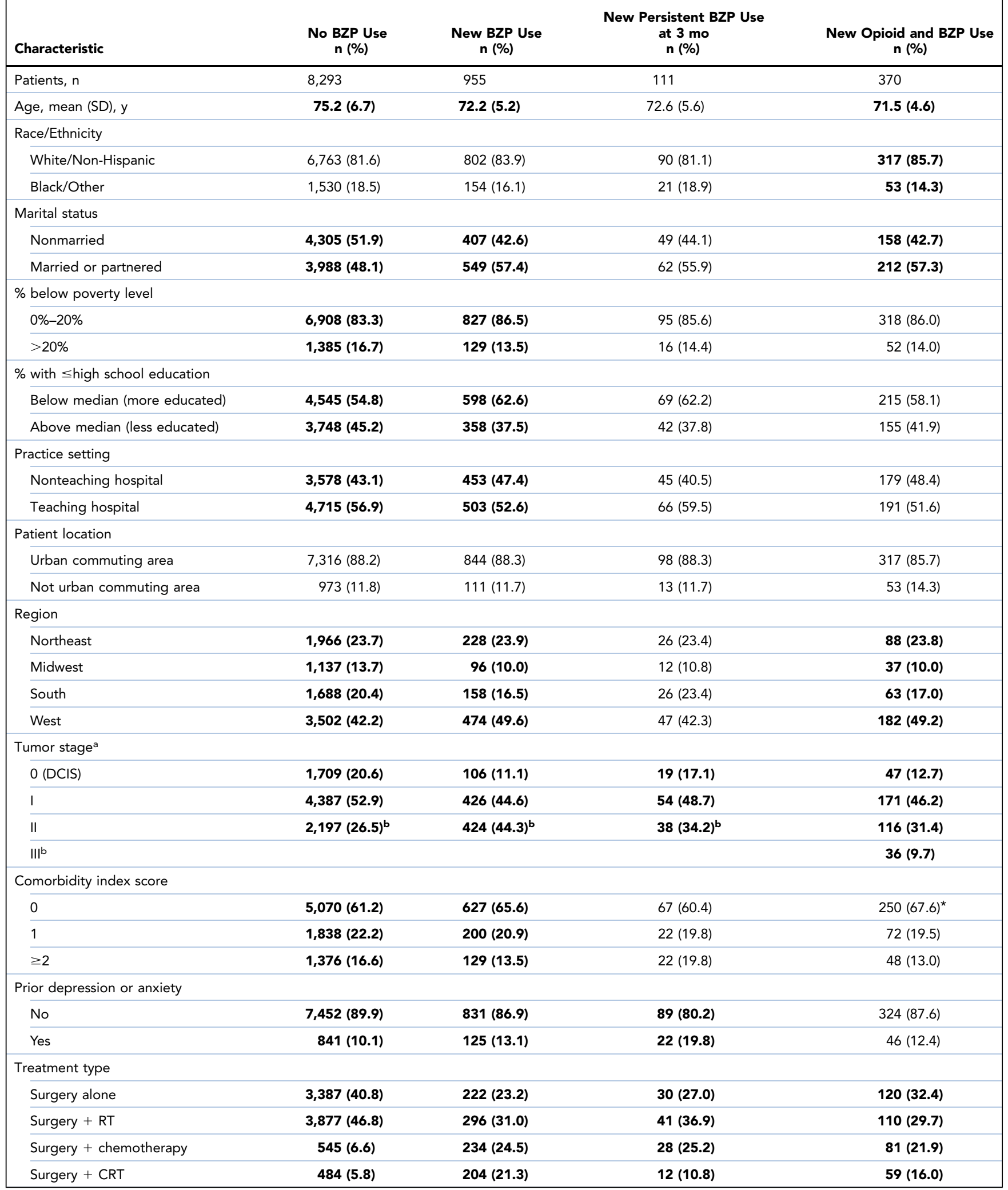




\section{eTable 2. Characteristics of Opioid- and Benzodiazepine-Naïve Patients (cont.)}

\begin{tabular}{|c|c|c|c|c|}
\hline Characteristic & $\begin{array}{c}\text { No BZP Use } \\
n(\%)\end{array}$ & $\begin{array}{c}\text { New BZP Use } \\
\text { n (\%) }\end{array}$ & $\begin{array}{c}\text { New Persistent BZP Use } \\
\text { at } 3 \text { mo } \\
n(\%)\end{array}$ & $\begin{array}{c}\text { New Opioid and BZP Use } \\
n(\%)\end{array}$ \\
\hline \multicolumn{5}{|c|}{ Endocrine therapy type } \\
\hline \multicolumn{5}{|c|}{ Aromatase inhibitors } \\
\hline No & 3,298 (39.8) & $311(32.5)$ & $42(37.8)$ & $116(31.4)$ \\
\hline Yes & $4,995(60.2)$ & 645 (67.5) & $69(62.2)$ & $254(68.6)$ \\
\hline \multicolumn{5}{|l|}{ Tamoxifen } \\
\hline No & $7,085(85.4)$ & $840(87.9)$ & $88(79.3)$ & $323(87.3)$ \\
\hline Yes & $1,208(14.6)$ & $116(12.1)$ & $23(20.7)$ & $47(12.7)$ \\
\hline \multicolumn{5}{|c|}{ First BZP prescribed } \\
\hline Lorazepam & - & $461(48.2)$ & $39(35.1)$ & - \\
\hline Alprazolam & - & $275(28.8)$ & 37 (33.3) & - \\
\hline Diazepam & - & $159(16.6)$ & $15(13.5)$ & - \\
\hline Other ${ }^{c}$ & - & $61(6.4)$ & $20(18.1)$ & - \\
\hline
\end{tabular}

Bold indicates statistically significant estimates based on a $P$ value threshold of .05 on the univariate analysis.

Abbreviations: BZP, benzodiazepine; CRT, chemoradiation therapy; DCIS, ductal carcinoma in situ; RT, radiation therapy.

aStaging based on the 6th edition of AJCC Cancer Staging Manual.

bStages II and III were combined to ensure cell size $>11$ for patient confidentiality.

Includes a small number of patients who received $>1$ BZP; however, numbers were too small to report combination use at 3 month.

${ }^{\star} P=.05-.06$. 